\title{
Photonic crystals of core-shell colloidal particles
}

\author{
Krassimir P. Velikov, ${ }^{a, \S)}$ Alexander Moroz, ${ }^{a}$ and Alfons van Blaaderen ${ }^{a, b, \S)}$ \\ a Physics and Chemistry of Condensed Matter, Debye Institute, Utrecht University, \\ Princetonlaan 5, 3584 CC Utrecht, The Netherlands \\ ${ }^{\mathrm{b}}$ FOM Institute for Atomic and Molecular Physics, Kruislaan 407, 1098 SJ \\ Amsterdam, The Netherlands
}

\begin{abstract}
We report on the fabrication and optical transmission studies of thin three-dimensional (3D) photonic crystals of high-dielectric $\mathrm{ZnS}$-core and low-dielectric $\mathrm{SiO}_{2}$-shell colloidal particles. These samples were fabricated using a vertical controlled drying method. The spectral position and width of a stopgap depend on the core-to-shell ratio, in a manner consistent with numerical calculations. Both experiments and calculations show that the relative L-stopgap width in the case of high-index core low-index shell particles can be larger in comparison to the case of homogeneous particles of either material. The core-shell morphology gives additional control over the photonic stopgap characteristics.
\end{abstract}

\footnotetext{
${ }^{\S}$ Corresponding authors; electronic mails: K.P.Velikov@phys.uu.nl, A.vanBlaaderen@phys.uu.nl
} 
Photonic crystals are materials with a periodically modulated dielectric constant. ${ }^{1,2}$ In analogy to electrons in a semiconductor, electromagnetic wave propagation in a photonic crystal can be inhibited for a certain frequency range resulting in the formation of a photonic bandgap. A promising way for fabrication of photonic crystals at optical wavelengths is the use of colloidal particles. ${ }^{3}$ Colloids can self-organize into a three-dimensional (3D) facecentered-cubic $(f c c)$ (or body-centered-cubic) crystal with a long-range periodicity. Although $f c c$ structures of dielectric spheres only possess stopgaps, ${ }^{4,5}$ the (local) density of states can still be manipulated significantly. ${ }^{6}$ Even for quite modest index contrast an interesting photonic switch can be realized with $f c c$ crystals. ${ }^{7}$ Photonic properties of colloidal crystals can be further improved by using a core-shell particle morphology. The latter can be used to enhance nonlinearities ${ }^{8}$ and to engineer photonic bandgap properties. ${ }^{3}$ Indeed, the full vector calculations on an $f c c$ crystal of core-shell particles have shown that the relative L-stopgap width $\left(\mathrm{g}_{w}\right)$ can be increased by more than $50 \%$ compared to the case of homogeneous particles. $^{5}$

Zinc sulfide $(\mathrm{ZnS})$, due to its high bulk refractive index $\left(\beta-\mathrm{ZnS} n_{589}=2.36\right)$ and lack of absorption in the visible and near IR region, ${ }^{9}$ is a convenient material for photonic applications. Spherical ZnS particles can be made with a wide range of sizes $(100-1500 \mathrm{~nm}$ in radius) and high monodispersity. ${ }^{10,11}$ Recently, we have shown that a silica layer can be deposited on the $\mathrm{ZnS}$ spheres, or vice versa, in order to change the filling fraction of the highindex material and tune the optical properties of the particles. ${ }^{11}$ Moreover, $\mathrm{ZnS}$ can be doped, e.g., with manganese, ${ }^{12}$ to induce luminescence, or, a fluorescent dye can be incorporated into the silica layer at a well-defined radial position. ${ }^{13}$ In addition, pure silica or pure $\mathrm{ZnS}$ particles, or shells of these materials, can be used for dielectric doping of photonic crystals in order to create localized modes inside the bandgap. ${ }^{14}$

In this letter, we demonstrate the fabrication of photonic crystals through a controlled 
drying $^{15,16}$ of a suspension of particles with a high-dielectric $\mathrm{ZnS}$ core and a low-dielectric $\mathrm{SiO}_{2}$ shell. We study optical transmission at normal incidence on thin photonic crystals. In accordance with previous calculations, ${ }^{5}$ we demonstrate that photonic crystals of high-index core and low-index shell particles posses a larger relative L-stopgap width in comparison to crystals of low-index core and high-index shell ${ }^{17}$ or homogeneous particles.

Photonic crystals were fabricated of monodisperse colloidal (core-shell) particles. Three different types of colloidal particles were used. Pure silica particles of radius $123 \mathrm{~nm}$ (with a relative width of the size distribution, $\delta=5 \%$ ) were synthesized following the Stöber-FinkBohn method. ${ }^{18}$ Homogeneous $\mathrm{ZnS}-\mathrm{SiO}_{2}$ composite particles of radius $125 \mathrm{~nm}(\delta=6 \%)$ were obtained after condensation of silica inside the pores of the $\mathrm{ZnS}$ spheres. In this case, micropores are filled up with silica, which results in an increase of the effective refractive index of the particle. $\mathrm{ZnS}$-core- $\mathrm{SiO}_{2}$-shell particles of total radius $128 \mathrm{~nm}(\delta=5 \%)$ with a $\mathrm{ZnS}-\mathrm{SiO}_{2}$ composite core radius of $84 \mathrm{~nm}(\delta=6 \%)$ were obtained after coating of $\mathrm{ZnS}$ cores with silica. The synthesis and optical characterization of the core-shell particles from $\mathrm{ZnS}$ and $\mathrm{SiO}_{2}$ are described elsewhere. ${ }^{11}$ The size and polydispersity were determined by transmission electron microscopy (TEM). The wavelength dependent effective refractive index of the $\mathrm{ZnS}$ was determined from extinction measurements of a dilute suspension in ethanol. ${ }^{11}$ Thin colloidal crystals were grown onto glass substrates using a vertical controlled drying method. ${ }^{15,16}$ This method allows the growth of large $(\mathrm{cm})$ arrays of colloidal crystals with precise control over the crystal thickness. Depending on the particle polydispersity, large (mm) single crystalline domains can be obtained. Scanning electron microscopy (SEM) was used to determine the number of layers in the crystal. The optical transmission spectra, measured with a Cary 500 UV-near-IR spectrometer, were taken along the [111] crystallographic axis, normal to the direction of the glass plate. The light beam spot was about $5 \mathrm{~mm}^{2}$, which is comparable to the area of a single crystalline domain. 
Figure 1 shows SEM micrographs of thin planar crystals of close-packed monodisperse $\mathrm{ZnS}$-core-SiO ${ }_{2}$-shell colloidal particles. Cracks, typically observed every 5-10 $\mu \mathrm{m}$, are formed after drying and shrinkage of the particles. More cracks form under influence of the vacuum and electron beam in the SEM, therefore there are less cracks present in the films used for extinction measurements. However, the crystalline order extends over a much longer distance. The inset shows a TEM image of a single particle, where the $\mathrm{ZnS}$ core and $\mathrm{SiO}_{2}$ shell can be directly seen. Figure 2 shows optical transmission spectra of thin photonic crystals of $\mathrm{ZnS}-$ core- $\mathrm{SiO}_{2}$-shell particles. The spectra exhibit a minimum in the optical transmission, where the light satisfies the Bragg condition and is diffracted away from the propagation direction. The small shift $(\sim 7 \mathrm{~nm})$ of the stop bandgap position to longer wavelengths is a finite-size effect. The thickness dependence of the stopgap position disappears if the sample is thicker than $\sim 8$-10 layers. ${ }^{19}$ For the given dielectric contrast, this is also expected from onedimensional calculations. ${ }^{20}$ The fast decrease of the optical transmission, near, but before the absorption edge of $\mathrm{ZnS},{ }^{9}$ is due to the strong incoherent scattering from the crystal defects. Theoretical spectra were calculated using the layer KKR method. ${ }^{21}$ In order to compare with the numerical calculations, the background scattering was subtracted. ${ }^{19}$ The peak position, the width, and the interference ripples agree well with theory (Fig. 2(b)). However, the height of the maximum does not. This is because of the unavoidable presence of crystal defects. An exact match of the experimental spectra is difficult to obtain, because the crystal thickness is very likely not constant over the measured region.

In order to demonstrate the importance of the particle morphology we performed calculations for infinite crystals. Figure 3 shows the calculated relative L-stopgap width, $\mathrm{g}_{w}$, of $\mathrm{ZnS}$-core-SiO${ }_{2}$-shell and $\mathrm{SiO}_{2}$-core- $\mathrm{ZnS}$-shell particles as a function of the core-to-totalradius ratio $\left(\gamma=R_{c} / R\right)$. The calculations were performed using a wavelength dependent effective refractive index. ${ }^{11}$ In both cases, the filling fraction of bulk $\mathrm{ZnS}$ (in a silica matrix, $n$ 
$=1.45$ ) in the particle core or shell was 0.62 . The high-index core low-index-shell particles display a maximum in the $\mathrm{g}_{w}$ at $\gamma \sim 0.70$, which is about $20 \%$ larger than that of a photonic crystal of homogeneous particles with the higher refractive index. It is remarkable that calculations show that a so-called air-sphere crystal, with the high index material surrounding a sphere of air, also has a smaller $\mathrm{g}_{w}$ compared to the case of $\gamma \sim 0.70$. In contrast, the reverse system displays a minimum at $\gamma \sim 0.76$ and a gap width that is lower than that of homogeneous particles. These calculations demonstrate the effect of the morphology and optimization of the filling fraction of the high dielectric material, using core-shell particles, on the optical properties of photonic crystals. For the L-stopgap the high-index core low-index shell morphology is clearly better than the low-index-core high-index shell ${ }^{17}$ for photonic applications. Figure 4 compares measured optical transmission spectra of thin photonic crystals from silica, $\mathrm{ZnS}$-core- $\mathrm{SiO}_{2}$-shell, and homogeneous $\mathrm{ZnS}_{-} \mathrm{SiO}_{2}$ composite particles of similar radii. Despite being of almost the same total radius, the L-stopgap moves towards longer wavelengths because of the increased dielectric contrast. Figure 5 presents the experimental and calculated relative L-stopgap width, $\mathrm{g}_{w}$, measured at half-maximum as a function of the core-to-total-radius ratio. The values determined from the experimental spectra (Fig. 4) are in good agreement with those from the theoretical spectra for an eight-layer thick crystal. However, the $\mathrm{g}_{w}$ obtained from the experiment is slightly broadened due to the presence of a moderate disorder in the crystal. This effect has been observed earlier. ${ }^{22}$ Assuming equal contributions of the crystal defects in the three cases, based on the similar particle polydispersity, a clear maximum can be distinguished in the case of the crystal of core-shell particles. One should notice that the $\mathrm{g}_{w}$ for thin crystals is still strongly dependent on the number of crystal layers. For instance, calculations for seven layers showed a $\sim 1 \%$ increase of $g_{w}$.

In conclusion, we have demonstrated the fabrication of photonic crystals of high-index 
$\mathrm{ZnS}$ core and low-index $\mathrm{SiO}_{2}$ shell colloidal particles. Due to the optimal filling fraction of the high dielectric material, photonic crystals from core-shell particles possess a larger relative L-stopgap width than crystals from homogeneous particles. By applying a lowdielectric coating we demonstrated the ability to control the filling fraction of the high dielectric material and optical properties of the photonic crystal. The combination of $\mathrm{ZnS}$ and $\mathrm{SiO}_{2}$ opens new possibilities to study dielectrically and fluorescently doped photonic crystals.

We would like to thank A. Imhof, J. P. Hoogenboom (AMOLF), E. J. Vlietstra, and C. M. van Kats (Utrecht University) for their assistance and helpful discussions. This work is part of the research program of the 'Stichting voor Fundamenteel Onderzoek der Materie', which is financially supported by the 'Nederlandse Organisatie voor Wetenschappelijk Onderzoek'. 


\section{References}

${ }^{1}$ E. Yablonovitch, Phys. Rev. Lett. 58, 2059 (1987).

${ }^{2}$ V. P. Bykov, Sov. J. Quantum Electron. 4, 861 (1975).

${ }^{3}$ A. van Blaaderen, MRS Bull. 23, 39 (1998).

${ }^{4}$ H. S. Sozuer, J. W. Haus, and R. Inguva, Phys. Rev. B-Condens Matter 45, 13962 (1992).

${ }^{5}$ A. Moroz and C. Sommers, J. Phys.-Condes. Matter 11, 997 (1999).

${ }^{6}$ Z. Zhang and S. Satpathy, Phys. Rev. Lett. 65, 2650 (1990).

${ }^{7}$ G. S. Pan, R. Kesavamoorthy, and S. A. Asher, Phys. Rev. Lett. 78, 3860 (1997).

${ }^{8}$ D.S. Chemla and D.A. Miller, Opt. Lett., 522 (1986).

${ }^{9}$ S. M. Scholz, R. Vacassy, J. Dutta et al., J. Appl. Phys. 83, 7860 (1998).

${ }^{10}$ D. M. Wilhelmy and E. Matijevic, J. Chem. Soc. Faraday Trans. 80, 563 (1984).

${ }^{11}$ K. P. Velikov and A. van Blaaderen, to appear in Langmuir, (2001).

${ }^{12}$ R.N. Bhargava, D. Gallagher, X. Hong et al., Phys. Rev. Lett. 72, 416 (1994).

${ }^{13}$ A. van Blaaderen and A. Vrij, Langmuir 8, 2921 (1992).

${ }^{14}$ R.D. Pradhan, I.I. Tarhan, and G.H. Watson, Phys. Rev. B-Condens Matter 54, 13721 (1996).

${ }^{15}$ N.D. Denkov, O.D. Velev, P.A. Kralchevsky et al., Langmuir 8, 3183 (1992).

${ }^{16}$ P. Jiang, J. F. Bertone, K. S. Hwang et al., Chem. Mat. 11, 2132 (1999).

${ }^{17}$ M. L. Breen, A. D. Dinsmore, R. H. Pink et al., Langmuir 17, 903 (2001).

${ }^{18}$ W. Stober, A. Fink, and E. Bohn, J. Colloid Interface Sci. 26, 62 (1968).

${ }^{19}$ J. F. Bertone, P. Jiang, K. S. Hwang et al., Phys. Rev. Lett. 83, 300 (1999).

${ }^{20}$ C. Rorres, SIAM J. Appl. Math., 303 (1974).

${ }^{21}$ N. Stefanou, V. Yannopapas, and A. Modinos, Comput. Phys. Commun. 113, 49 (1998).

22 Y. A. Vlasov, M. Deutsch, and D. J. Norris, Appl. Phys. Lett. 76, 1627 (2000). 


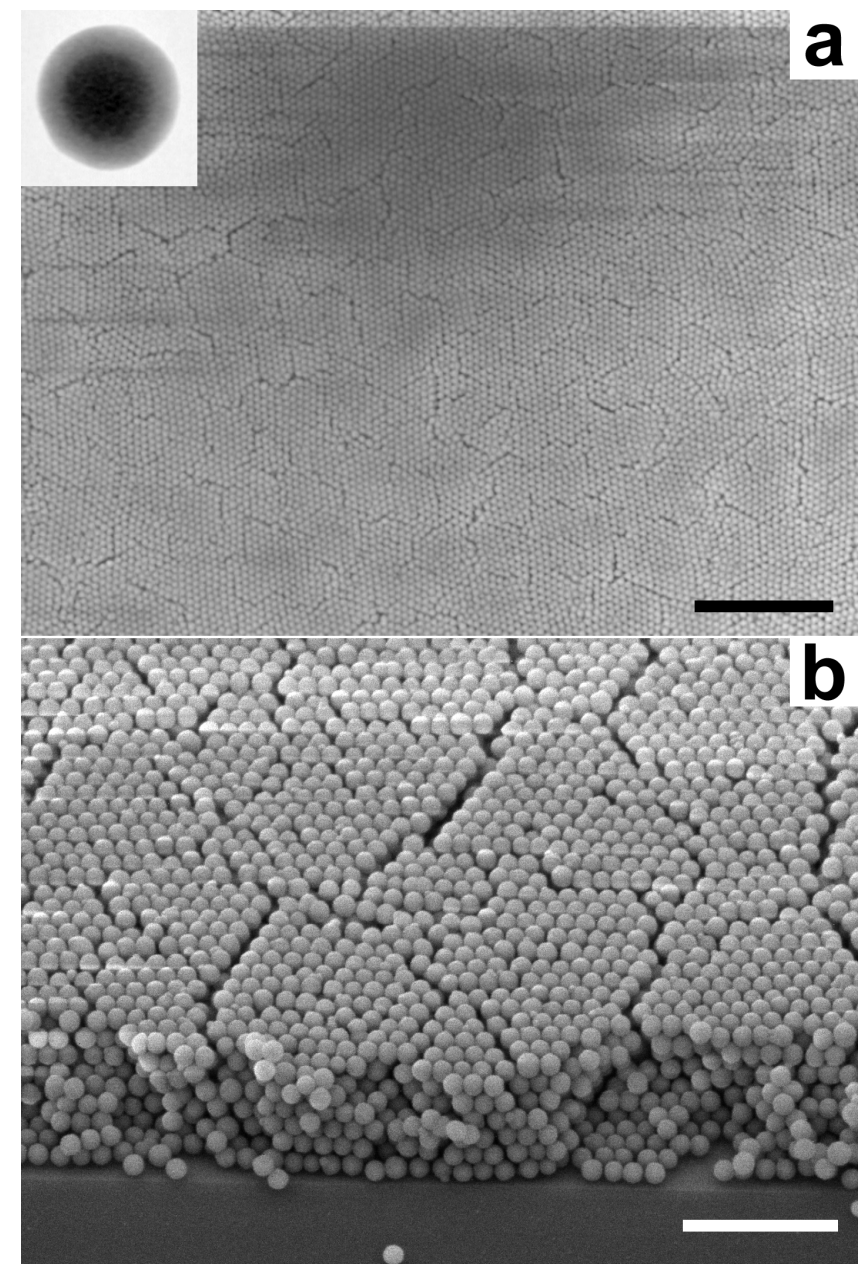

FIG. 1. Scanning electron micrographs (SEM) of a planar photonic crystal of close-packed core-shell colloidal particles. (a) Top view of the crystal showing a [111]-crystal plane. The scale bar is $5 \mu \mathrm{m}$. The inset shows a transmission electron micrograph (TEM) of a single $\mathrm{ZnS}$ core- $\mathrm{SiO}_{2}$-shell particle. The total particle radius is $128 \mathrm{~nm}$ with a $44 \mathrm{~nm}$ silica shell. (b) Side view of the crystal. The scale bar is $2 \mu \mathrm{m}$. 


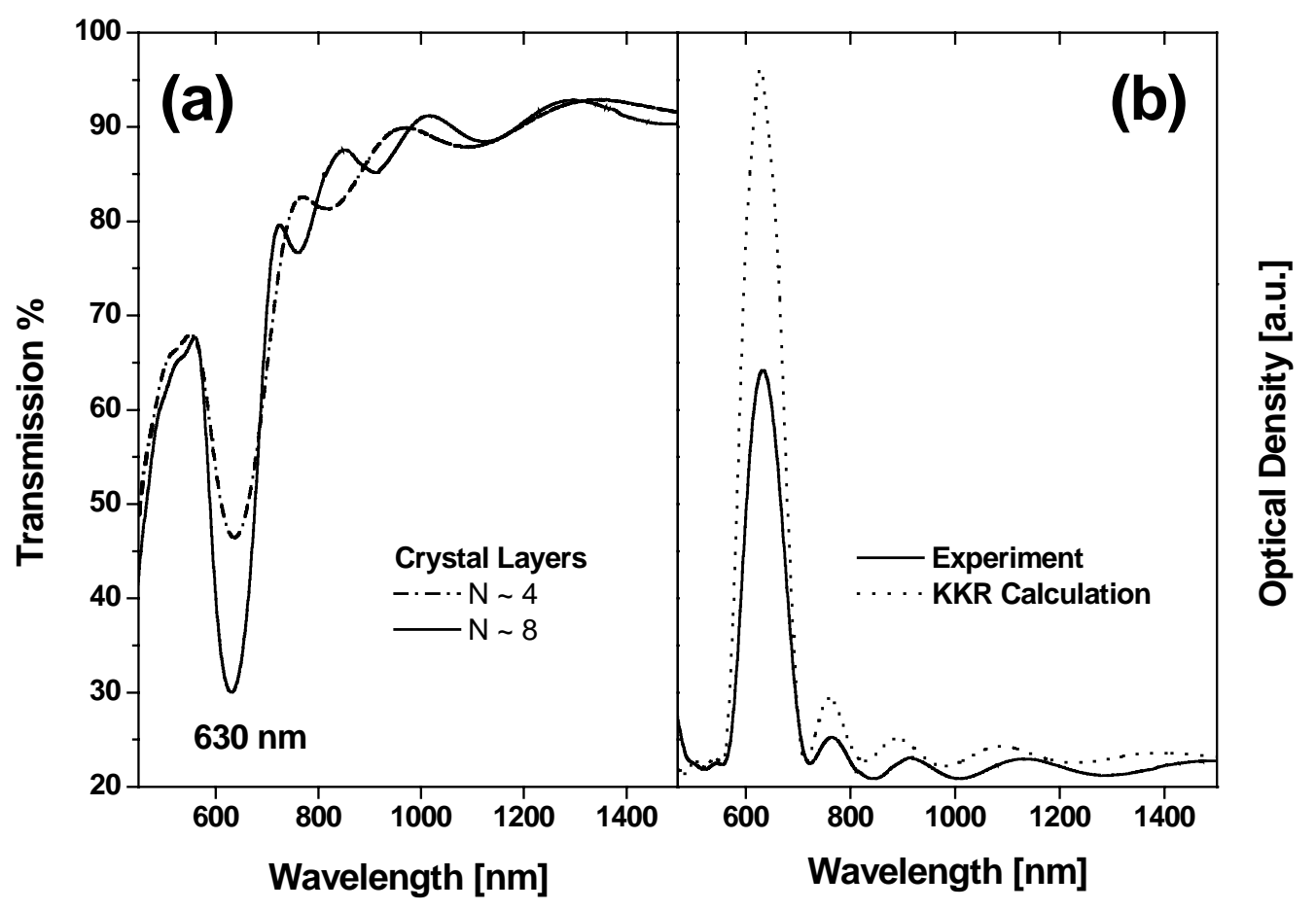

FIG. 2 Optical transmission spectra (a) measured on photonic crystals of $\mathrm{ZnS}$-core-SiO ${ }_{2}$-shell colloidal particles grown on glass substrates. The experimental spectra were taken along the [111] crystallographic axis. (b) Calculated (dotted line) and experimentally determined (solid line) optical density spectra of an eight-layer thick crystal on a glass substrate $\left(n_{\text {sub }}=1.40\right)$. The theoretical spectrum was calculated using the parameters similar to those determined from light scattering and TEM $\left(R_{\mathrm{c}}=93.4 \mathrm{~nm}, R=130 \mathrm{~nm}, \varphi_{\mathrm{ZnS}}=62 \%, n_{589}=1.92\right)$. 


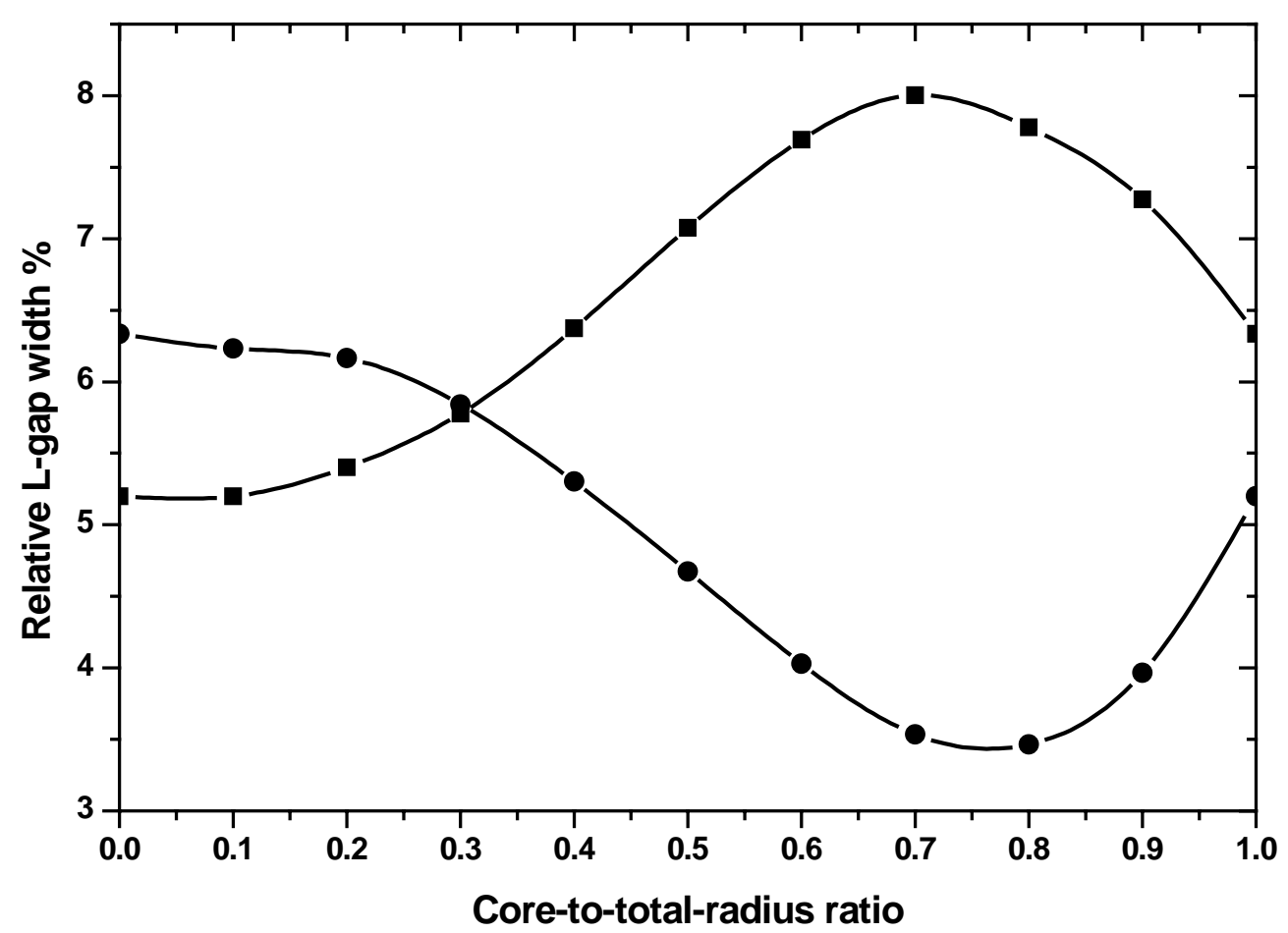

FIG. 3 Calculated relative L-stopgap width as a function of the core-to-total-radius ratio for an infinite crystal of high-index core low-index shell particles (squares) and, for comparison, the reverse structure with the same parameters (circles). The high-index core (shell) consists of $62 \%$ bulk $\mathrm{ZnS}$ embedded in a silica matrix. The low-index shell (core) consists of pure silica. The wavelength dependent effective dielectric constant of the high-index core (shell) was calculated using the Maxwell-Garnett formula. ${ }^{11}$ The line is to guide the eye. 


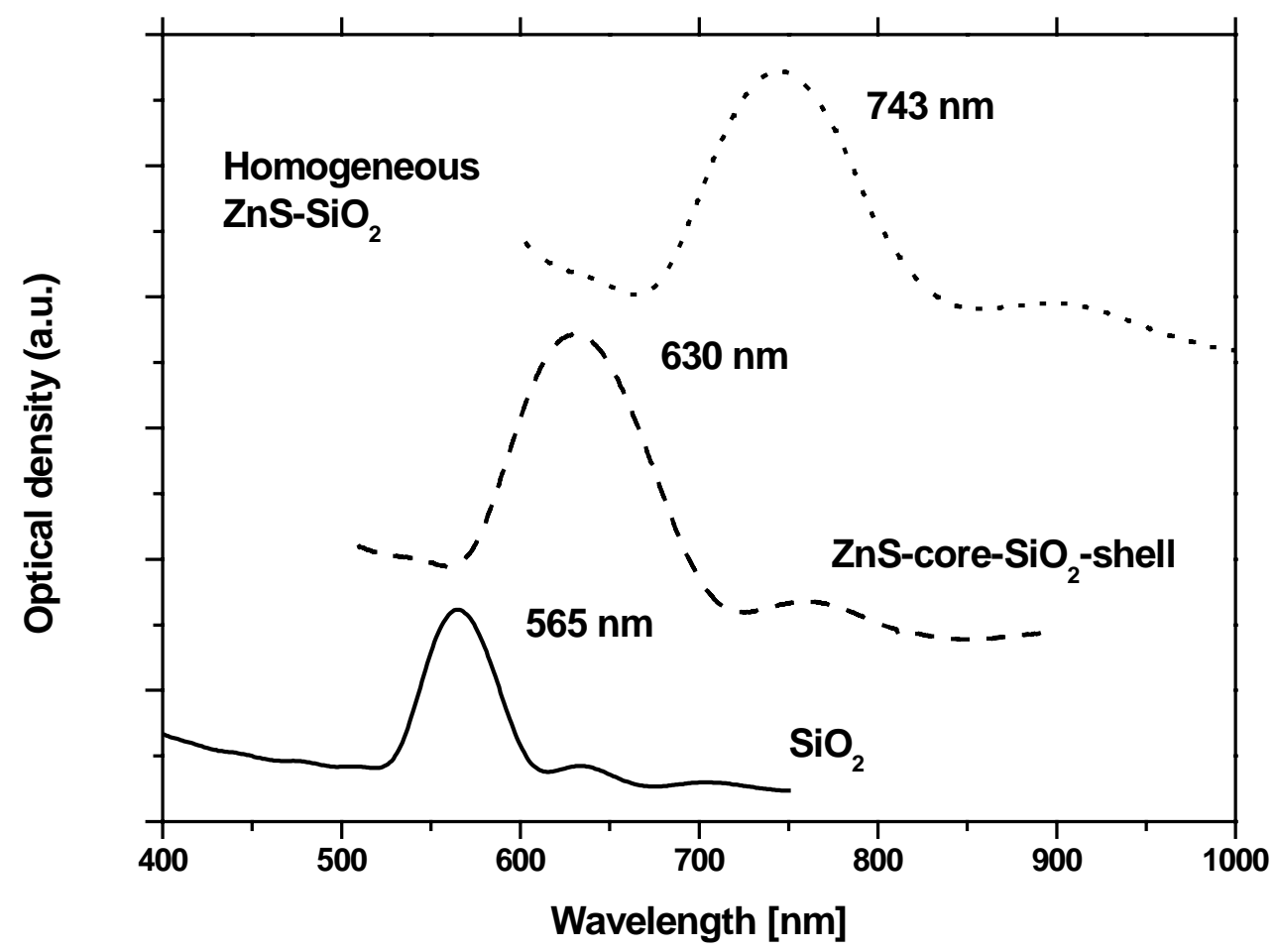

FIG. 4. Optical density (OD) spectra measured on photonic crystals of homogeneous ZnS$\mathrm{SiO}_{2}$ composite (dotted line), $\mathrm{ZnS}$-core- $\mathrm{SiO}_{2}$-shell (dashed line), and pure silica (solid line) particles. The average crystal thickness is eight layers. The position of the L-stopgap shifts towards longer wavelengths as the effective refractive index of the single particle increases. 


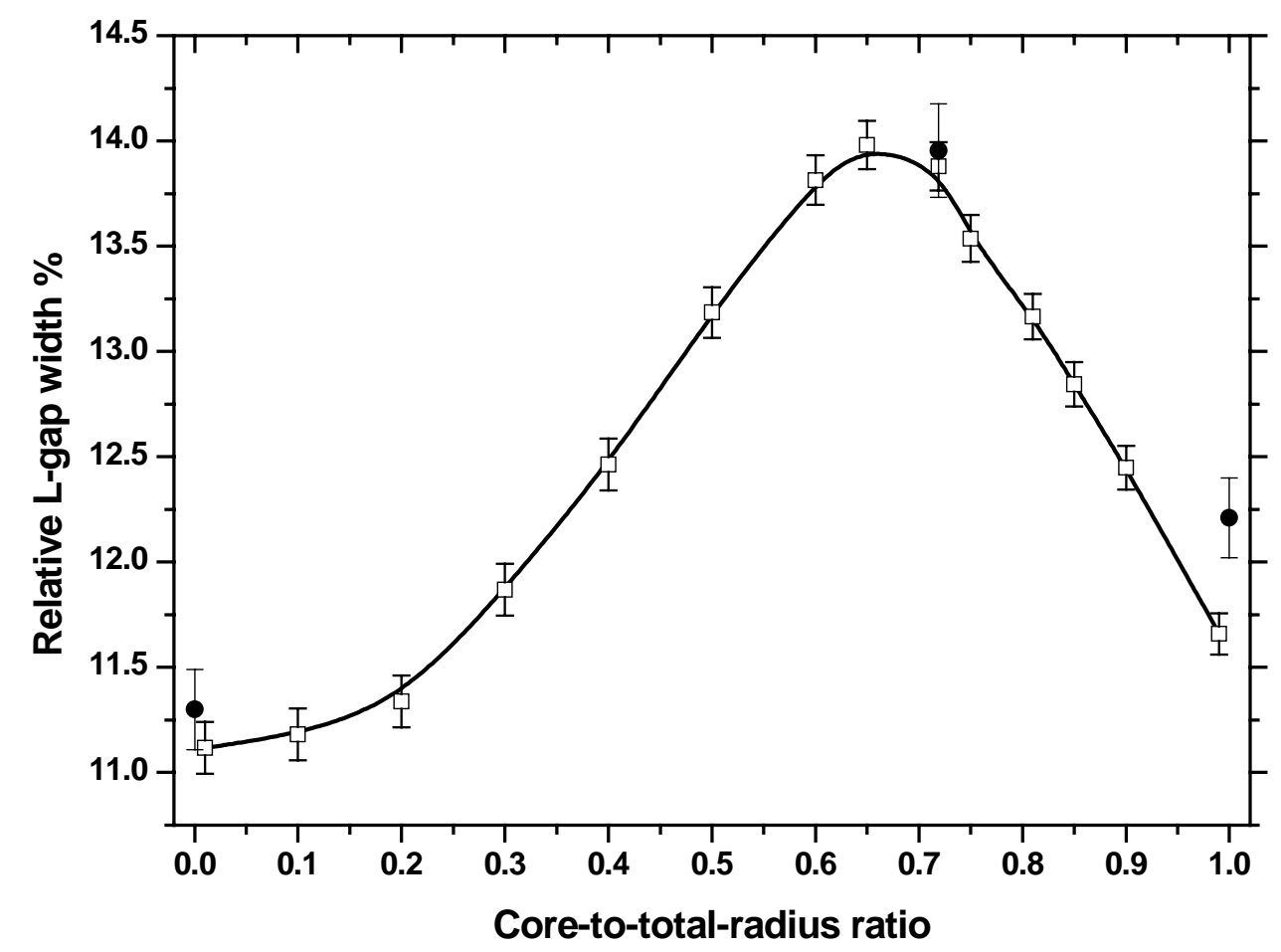

FIG. 5. Relative L-stopgap width at half-maximum determined from the experimental (circles) and theoretical (open squares) spectra as a function of the core-to-total-radius ratio for eight-layers thick crystals. The experimental data are obtained from crystals of pure silica, $\mathrm{ZnS}$-core-SiO${ }_{2}$-shell, and $\mathrm{ZnS}-\mathrm{SiO}_{2}$ composite particles of similar radii (Fig. 4). Theoretical spectra were calculated with the same particle parameters as in Fig. 1(b). There is an optimal ratio $(\gamma \sim 0.65)$ at which the stopgap has a maximal value. The line is to guide the eye. 